Comyns, Thomas M, Flanagan, Eamonn

P, Fleming, Sean, Fitzgerald, Evan and Harper, Damian (2019) Inter-Day Reliability and Usefulness of Reactive Strength Index Derived From Two Maximal Rebound Jump Tests. International Journal of Sports Physiology and Performance. pp. 1-17.

Downloaded from: http://ray.yorksj.ac.uk/id/eprint/3770/

The version presented here may differ from the published version or version of record. If you intend to cite from the work you are advised to consult the publisher's version: http://dx.doi.org/10.1123/ijspp.2018-0829

Research at York St John (RaY) is an institutional repository. It supports the principles of open access by making the research outputs of the University available in digital form. Copyright of the items stored in RaY reside with the authors and/or other copyright owners. Users may access full text items free of charge, and may download a copy for private study or non-commercial research. For further reuse terms, see licence terms governing individual outputs. Institutional Repository Policy Statement

\title{
RaY
}

Research at the University of York St John

For more information please contact RaY at ray@yorksj.ac.uk 


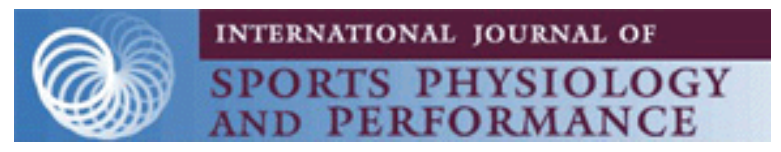

\section{Inter-day reliability and usefulness of reactive strength index derived from two maximal rebound jump tests}

\begin{tabular}{|r|l|}
\hline Journal: & International Journal of Sports Physiology and Performance \\
\hline Manuscript ID & IJSPP.2018-0829.R1 \\
\hline Manuscript Type: & Original Investigation \\
\hline Author: & 28-Jan-2019 \\
\hline Complete List of Authors: & $\begin{array}{l}\text { Comyns, Tom; University of Limerick, PESS } \\
\text { Flanagan, Eamonn; Sport Ireland Institute, Strength and conditioning } \\
\text { Fleming, Sean; University of Limerick, PESS } \\
\text { Ftizgerald, Evan; University of Limerick, PESS } \\
\text { Harper, Damian; York St John University, School of Sport }\end{array}$ \\
\hline Keywords: & $\begin{array}{l}\text { performance testing, strength and conditioning, strength testing, } \\
\text { stretch-shortening cycle, plyometrics }\end{array}$ \\
\hline &
\end{tabular}

\section{SCHOLARONE ${ }^{m}$ Manuscripts}




\section{Inter-day reliability and usefulness of reactive strength index derived from two maximal rebound jump tests.}

Submission type: Original Investigation

Thomas M Comyns ${ }^{1,2}$, Eamonn P Flanagan ${ }^{3}$, Sean Fleming ${ }^{1}$, Evan Fitzgerald ${ }^{1}$ and Damian J Harper $^{4}$

${ }^{1}$ Department of Physical Education and Sport Sciences, University of Limerick, Limerick, Ireland.

${ }^{2}$ Health Research Institute, University of Limerick, Limerick, Ireland.

${ }^{3}$ Sport Ireland Institute, IIS Building, National Sports Campus, Abbotstown, Dublin 15, Ireland.

${ }^{4}$ School of Sport, York St John University, York, United Kingdom.

\section{Corresponding Author:}

Tom Comyns,

Department of Physical Education and Sports Sciences,

University of Limerick,

Limerick,

Ireland

Email: tom.comyns@ul.ie

Telephone: +353 863617298

Preferred running head: Reliability and usefulness of reactive strength index

Abstract word count: 217

Text-only word count: $\mathbf{2 , 7 4 8}$

Number of References: 27

Number of Figures: 2

Number of Tables: 2 
Purpose: This investigation examined the inter-day reliability and usefulness of reactive strength index (RSI) derived from a maximal 5 rebound jump test ( $5_{\max }$ RJT) and a maximal 10 rebound jump test (10/5 RJT). Methods: Twenty male field sport athletes $(24.5 \pm 3.0 \mathrm{y} ; 1.78 \pm 0.1 \mathrm{~m} ; 84.9 \pm 5.2 \mathrm{~kg})$ performed 2 maximal repetitions of the $5_{\max }$ RJT and the $10 / 5$ RJT on two testing days following a specific warm up. A one week period separated each testing day and these sessions were proceeded by a familiarisation session. RSI was calculated by dividing jump height (m) by contact time (s). The $5_{\max }$ RJT and the 10/5 RJT trial with the highest RSI on each testing day was used for reliability and usefulness analysis. Results: Both tests were deemed reliable for determining RSI for male, female and pooled male and female cohorts as the ICCs $\geq 0.80$ and the $\mathrm{CV} \leq 10 \%$. Only the $5_{\max }$ RJT was rated as 'good' at detecting the smallest worthwhile change (SWC) in performance for female athletes (SWC: $0.10>$ TE:0.07). The $5_{\max }$ RJT for males and the 10/5 RJT for males and females were rated as 'good' in detecting a moderate change in performance only. Conclusions: Both tests are reliable for the determination of RSI but the usefulness of the tests in detecting the SWC is questionable.

Keywords: performance testing, strength and conditioning, strength testing, stretchshortening cycle, plyometrics 
Sport demands individuals to acquire and execute a vast repertoire of movement skills. A fundamental requirement to safe and efficient execution of these movement skills is the identification, development and assessment of specific strength (dynamic, isometric, reactive) qualities. ${ }^{1}$ Reactive strength evaluates the athlete's ability to efficiently brake and absorb (eccentric) forces within specific time frames, before subsequently generating a propulsive (concentric) force. ${ }^{2}$ This has also been recognized as an individual's stretch load tolerance. ${ }^{3}$ Since these qualities represent the efficiency of an athlete's stretch-shortening cycle (SSC) capabilities, testing and monitoring this strength quality has been of significant interest to researchers and practitioners for some time. ${ }^{3}$

Self-regulated repetitive vertical hopping or continuous rebound jump tests provide a simple and controlled way to evaluate neuromuscular properties and muscle-tendon unit mechanics fundamental to fast-SSC (ground contact times (GCT); $\leq 0.25 \mathrm{~s}$ ) performance. ${ }^{4}$ The reactive strength index (RSI) is frequently used to provide an indicator of fast-SSC capabilities. ${ }^{5}$ However, despite being extensively used to evaluate drop jump performance, there is limited research that has used RSI during continuous maximal rebound jumps ${ }^{6-12}$, with only two studies to date examining the reliability of these measures. ${ }^{13,14}$

Lloyd, et al. ${ }^{14}$ examined the within and between session reliability of a maximal 5-rebound jump ( $5_{\max }$ RJT) protocol in male youths and found that despite RSI having acceptable levels of test-retest reliability, the trial to trial variation (measurement error) in RSI scores was less reliable (coefficient of variation (CV): 11-21\%) making it difficult to detect small but meaningful changes in RSI performance. It was suggested that the large CV could be attributed to variations in GCT, arising from an inability to control loading forces during repeated ground interactions. Moresi, et al. ${ }^{15}$ has shown that through a process of data reduction 'atypical' scores in rebound jump can be excluded ensuring reliable trial-to-trial variation $(\mathrm{CV} \leq 10 \%)$ even for youth and less experienced individuals. Using a maximal 10 rebound jump protocol (10/5 RJT), Harper, et al. ${ }^{13}$ found that by removing the 5 lowest RSI scores across the 10 rebound jumps a CV of less than $10 \%$ could be obtained. Despite this, the usefulness of the 10/5 RJT test for detecting the smallest worthwhile change (SWC) was not evaluated.

Given the potential for rebound RSI jump performance to monitor changes in ankle joint stiffness particularly in the eccentric phase ${ }^{16}$, the capacity to sustain high eccentric muscle activity or reactive strength ${ }^{6,7}$, mechanical efficiency in runners ${ }^{17}$ and ankle joint kinetics associated with maximal velocity sprint running ${ }^{8}$, it is of significant interest to practitioners to establish and compare both noise (CV) and signal (SWC) in order to allow inferences to be made on the true magnitude of individual changes in reactive strength performance. ${ }^{18}$ Therefore, the aim of this study was to establish and compare the inter-day reliability and usefulness of the $5_{\max } \mathrm{RJT}$ and 10/5 RJT test for detecting practically small but important changes in RSI in both male and female team sport athletes. 
Methods

\section{Participants}

Twenty male (mean $\pm \mathrm{SD}, 24.5 \pm 3.0 \mathrm{y} ; 1.78 \pm 0.1 \mathrm{~m} ; 84.9 \pm 5.2 \mathrm{~kg}$ ) and fifteen females (mean $\pm \mathrm{SD}, 21.1 \pm 0.9 \mathrm{y} ; 1.65 \pm 0.73 \mathrm{~m} ; 62.0 \pm 5.1 \mathrm{~kg}$ ) from Gaelic games took part in this study. The male participants were elite level inter-county Hurling players and the females played Gaelic Football at a collegiate level. All had at least 6 months of resistance training experience and were familiar with bilateral vertical hopping and fast-SSC training. Prior to participation, subjects read and signed an informed consent and completed the Physical Activity Readiness Questionnaire (PAR-Q). All subjects answered 'No' to all questions on the PAR-Q. Approval for the study design was obtained prior to the commencement of the study from the University Institution Ethical Review Board, and all procedures were in accordance with the Declaration of Helsinki.

\section{Study Design}

A cross-sectional study design with repeated measures was used. All participants took part in two testing sessions where two trials of both the $5_{\max }$ RJT and the 10/5 RJT were completed on each testing day to assess inter-day RSI reliability. Prior to these testing sessions the participants completed a familiarisation session. The three testing days were separated by a one week period.

\section{Methodology}

All of the three sessions took place on the same day of the week and at the same time of the day to control for circadian variation. ${ }^{19}$ Both the familiarisation and the two testing sessions followed the same format. The sessions began with a warm up consisting of 5 minutes of low intensity jogging and lower limb dynamic stretches. Following this, the participants performed 2 sets of 5 double leg ankle jumps and were given 2 minutes of seated rest before the commencement of the jump testing.

Once the warm-up was complete, the participants completed 2 trials of the $5_{\max }$ RJT followed by 2 trials of the $10 / 5$ RJT. There was $60 \mathrm{~s}$ rest between each trial 13 and 2 minutes between each jump type. For both jump protocols, participants were instructed to keep hands on hips to avoid upper-body interference ${ }^{20}$, jump and land on the same spot, land with legs extended and then flex them and to look ahead at a fixed point at all times. The participants were also asked to maximise jump height and minimize ground contact time. ${ }^{14}$ Specifically they were instructed to imagine the ground is a hot surface, jump as high as possible and to imagine their leg is like a stiff spring rebounding off the ground'. ${ }^{5}$ The 5 max RJT involved the participants completing a countermovement jump followed by 4 maximal rebound jumps. The RSI value was calculated for each of the maximal rebound jumps by dividing jump height by ground contact time. ${ }^{3}$ The height jumped was defined as the flight time component and it was determined using the equation $\mathrm{HJ}=\left(9.81 \times \mathrm{FT}^{2}\right) / 8$ from Bosco, et al.. ${ }^{21}$ The average RSI of the 4 rebound jumps was subsequently determined to reflect the overall RSI value for this trial $\left(5_{\max }\right.$ RJT-RSI) and the trial with the highest RSI value was used for subsequent analysis. The 10/5 RJT involved the participants performing a countermovement jump followed by 10 maximal rebound jumps. RSI for each jump was calculated as described previously and the average of the 5 best RSI scores with GCT less than $0.25 \mathrm{~s}$ was used to determine an overall RSI value for 
this trial (10/5 RJT-RSI). ${ }^{13}$ Again the trial with the best RSI score was used for statistical analysis.

All $5_{\max }$ RJT and 10/5 RJT trials were measured using the Optojump ${ }^{\mathrm{TM}}$ (Microgate, Bolzano, Italy) system. The Optojump ${ }^{\mathrm{TM}}$ consists of two parallel bars connected to a personal computer with one bar acting as a transmitter unit containing 96 light emitting diodes positioned $0.003 \mathrm{~m}$ above the ground and the other bar acting as a receiver unit. ${ }^{22}$ When a participant performs a rebound jump within the parallel bar configuration the light is interrupted by the participant's foot during the jump, which triggers the timer in the unit and records with a precision of $1 \mathrm{~ms}^{22}$ The total time that the light is interrupted is a measure of contact time and the total time between interruptions is a measure of flight time. ${ }^{22}$ This system has been reported as a valid measurement of RSI. ${ }^{22}$

\section{Statistical Analyses}

The trial with the highest RSI score for both jump protocols on each testing day was used for inter-day reliability analysis, which was performed for the entire group and males and females separately. Assumption of normality for all data was confirmed using the Shapiro-Wilk statistic. Reliability was calculated by determining the coefficient of variation (calculated as the typical error and expressed as a CV) and the intraclass correlation coefficient (ICC) with 95\% confidence intervals (95\% CI) using a Microsoft Excel spreadsheet. ${ }^{23}$ Acceptable reliability was determined at an $\mathrm{ICC} \geq 0.8$ and $\mathrm{CV} \leq 10 \%{ }^{23}$

Usefulness was determined by comparing typical error (TE) to the SWC using a Microsoft Excel spreadsheet. ${ }^{23}$ The SWC was calculated by multiplying the between-subject $\mathrm{SD}$ by $0.2\left(\mathrm{SWC}_{0.2}\right)$, which represents a typical small effect and by $0.5\left(\mathrm{SWC}_{0.5}\right)$ which is an alternate moderate effect. In line with recommendations from Hopkins ${ }^{24}$, the test was rated as 'good' if the TE was below the SWC, as 'ok' if the TE was similar to the SWC and as 'marginal' in detecting meaningful change if the TE was higher than the SWC.

\section{Results}

Male and female highest (mean \pm SD) RSI scores for both RJT protocols for day one and day two are shown in Table 1. In Table 2 the results pertaining to the ICC, $\mathrm{CV}, \mathrm{TE}, \mathrm{SWC}_{0.2}$ and $\mathrm{SWC}_{0.5}$ are detailed. Figure 1 shows how the various cohorts meet the ICC criteria of $\geq 0.8$. Similarly figure 2 illustrates how these cohorts satisfied the CV criteria of $\leq 10 \%$.

The results pertaining to the usefulness of the $10 / 5$ RJT and the $5_{\max }$ RJT are detailed in Table 2. Only the $5_{\max }$ RJT in females was shown to be able to detect a 'small' worthwhile change in RSI. Both the 10/5 RJT and 5max RJT was rated as 'good' for detecting a 'moderate' change in RSI.

\section{Discussion}

Both the $5_{\max }$ RJT and the $10 / 5$ RJT can be deemed as reliable tests of fast-SSC reactive strength index for male, female and pooled groups of male and female field 
sport athletes. With respect to the reliability of RSI measurement, both tests demonstrated a CV of less than $10 \%$ in each cohort and an ICC of $\geq 0.8$.

Despite being a reliable test, the results of this study call into question the usefulness of the $5_{\max }$ RJT and the 10/5 RJT for this particular cohort of field sport athletes. The $10 / 5$ RJT had a typical error of 0.10-0.14 units for the measurement of RSI which is greater than the SWC for these male, female and pooled cohorts which ranged from 0.06-0.09 units. Thus, the efficacy of this test to detect the SWC is deemed to be marginal.

The $5_{\max }$ RJT had a lower typical error of $0.07-0.10$ units for the measurement of RSI. The SWC for these male, female and pooled cohorts ranged from 0.08-0.10 units. The $5_{\max }$ RJT demonstrated a good ability to detect the SWC in RSI in female athletes only. In the male cohort and the pooled cohort the efficacy of the test to detect the SWC was deemed to be marginal. Both tests were rated as "good" in terms of detecting moderate changes in reactive strength ( 0.5 effect size).

These results cast doubt as to the usefulness of the $5_{\max }$ RJT and the $10 / 5$ RJT as a daily or weekly monitoring tool due to its potential inability to detect the SWC. Weekly monitoring needs to be sensitive to small changes in physical condition in order to afford coaches the opportunity to manage training loads and optimise preparedness. However, the usefulness of any test may be dependent on the subject cohort's familiarity with the testing protocol. It could be expected that typical error of testing will reduce as subjects become more familiar (and more habituated) to a testing protocol.

In this cohort of subjects, the 10/5 RJT exhibited CVs of $7-10 \%$ and the $5_{\max }$ RJT demonstrated CV of 7-8\%. These are similar CVs as has previously been reported for reactive strength measurement by Beattie and Flanagan ${ }^{25}$ who observed CV of $8.5 \%$ for the drop jump from $40 \mathrm{~cm}$ (DJ-40) using a contact mat. In agreement with the current study, Beattie and Flanagan ${ }^{25}$ also observed that the DJ-40 reactive strength test was unable to detect the SWC. The observed CV was greater than the calculated SWC. However, other studies have observed much lower CV for reactive strength index in the drop jump. Markwick, et al. ${ }^{26}$ observed RSI CVs of $2.1-3.1 \%$ for basketball players in the drop jump across heights ranging from $20-50 \mathrm{~cm}$.

These conflicting results demonstrate that the "reliability" or usefulness of reactive strength testing may be population specific. For example, the population in the study by Markwick, et al. ${ }^{26}$ were professional basketball players. A reasonable expectation can be made that these subjects would have greater fast-SSC training experience than the current cohort of amateur field sport athletes. Although both studies utilized different reactive strength testing modalities, it is worth noting that the subjects utilized by Markwick, et al. ${ }^{26}$ demonstrated greater reactive strength ability than the subjects in the current study. The male professional basketball players in the study by Markwick et al. ${ }^{26}$ exhibited a mean RSI of 2.1 units compared to a mean RSI of $1.5-$ 1.6 units for males in the current study and a mean of 1.8 units for junior rugby players in the work of Beattie and Flanagan. ${ }^{25}$ 
224 It has also been demonstrated that there can be large variation in reliability between athletes within the same cohort and it has been recommended that individualised CV should be calculated to assess "meaningful change" on an athlete-by-athlete basis. ${ }^{25}$ By comparing the change in performance (the signal) relative to the test's inter-day $\mathrm{CV}$ value (the noise), practitioners can begin to make decisions about the 'meaningfulness of change' in that variable and make an objective judgement on the changes in physical preparedness ${ }^{27}$. If the change in an athlete's RSI is outside their individualised $\mathrm{CV}$ for the test (i.e. the signal $\geq$ noise), then coaches can be confident that the change is a 'worthwhile' increase or decrease in reactive strength ${ }^{24}$.

With more experienced athletes or with greater exposure to the testing protocol, it is possible that the "usefulness" of the test may improve. However, this study highlights that for male and female amateur field sport athletes the current protocols, apart from the $5_{\max }$ RJT for females, are not enough to derive "useful" data and may not be able to detect the SWC.

\section{Practical Applications}

Both the $10 / 5 \mathrm{RJT}$ and $5_{\max }$ RJT demonstrated good inter-day reliability and thus have potential to be used as a measure of an athlete's fast-SSC capabilities. In addition, identifying small meaningful changes in RSI is of significant interest to practitioners. The dataset is limited in this study as only the $5_{\max }$ RJT for females demonstrated an ability to detect SWC for this population group. Practitioners should calculate their own bespoke SWC / TE data to assess the usefulness of the test for their own population. Finally, this study does provide practitioners with examples of typical error for both the $5_{\max }$ RJT and the 10/5 RJT. The 10/5 RJT had a typical error of 0.10 -0.14 units and the $5_{\max }$ RJT had a typical error of $0.07-0.10$ units for the measurement of reactive strength index. These TE examples, however, may be specific to the population group in question.

\section{Conclusions}

Both the $5_{\max }$ RJT and the 10/5 RJT are reliable tests of fast-SSC reactive strength capabilities for male, female and combined male and female groups of field sport athletes. However, this study casts some doubt on the ability of these tests to detect the SWC in reactive strength in field sport athletes. The tests do have the potential to detect a moderate change in reactive strength within this cohort. 
1. Suchomel TJ, Nimphius S, Stone MH. The Importance of Muscular Strength 
18. Buchheit M. The Numbers Will Love You Back in Return-I Promise. Int J Sport Physiol. 2016;11(4):551-554.

19. Atkinson G, Reilly T. Circadian Variation in sports performance. Sports Med. 1996;21(4):292-312.

20. Lees A, Vanrenterghem J, De Clercq D. Understanding how an arm swing enhances performance in the vertical jump. J Biomech. 2004;37(12):19291940.

21. Bosco C, Luhtanen P, Komi PV. A simple method for measurement of mechanical power in jumping. European Journal of Applied Physiology and Occupational Physiology. 1983;50(2):273-282.

22. Healy R, Kenny IC, Harrison AJ. Assessing Reactive Strength Measures in Jumping and Hopping Using the Optojump (TM) System. J Hum Kinet. 2016;54(1):23-32.

23. Hopkins WG. Measures of reliability in sports medicine and science. Sports Med. 2000;30(1):1-15.

24. Hopkins WG. How to interpret changes in an athletic performance test. Sportscience. 2004;8(1):1-15.

25. Beattie K, Flanagan E. Establishing the reliability and meaningful change of the drop-jump reactive-strength index. Journal of Australian Strength and Conditioning. 2015;23(5):12-18.

26. Markwick WJ, Bird SP, Tufano JJ, Seitz LB, Haff GG. The Intraday Reliability of the Reactive Strength Index Calculated From a Drop Jump in Professional Men's Basketball. Int J Sport Physiol. 2015;10(4):482-488.

27. Coutts A, Cormack S. Monitoring the training response. In: Joyce D, Lewindon D, eds. High-performance training for sports. Leeds, United Kingdom: Human Kinetics; 2014:71-84. 
$340 \quad$ Figure Captions

341

342

343 Figure 1. ICCs $\pm 95 \%$ CI for both jump protocols for males, females and males and

344 females combined. Grey shaded area $=$ zone of acceptable reliability $($ ICC $\geq 0.8)$.

345

346

347 Figure 2. CVs $\pm 95 \%$ CI for both jump protocols for males, females and males and

348 females combined. Grey shaded area $=$ zone of acceptable reliability $(\mathrm{CV} \% \leq 10 \%)$. 349

350 


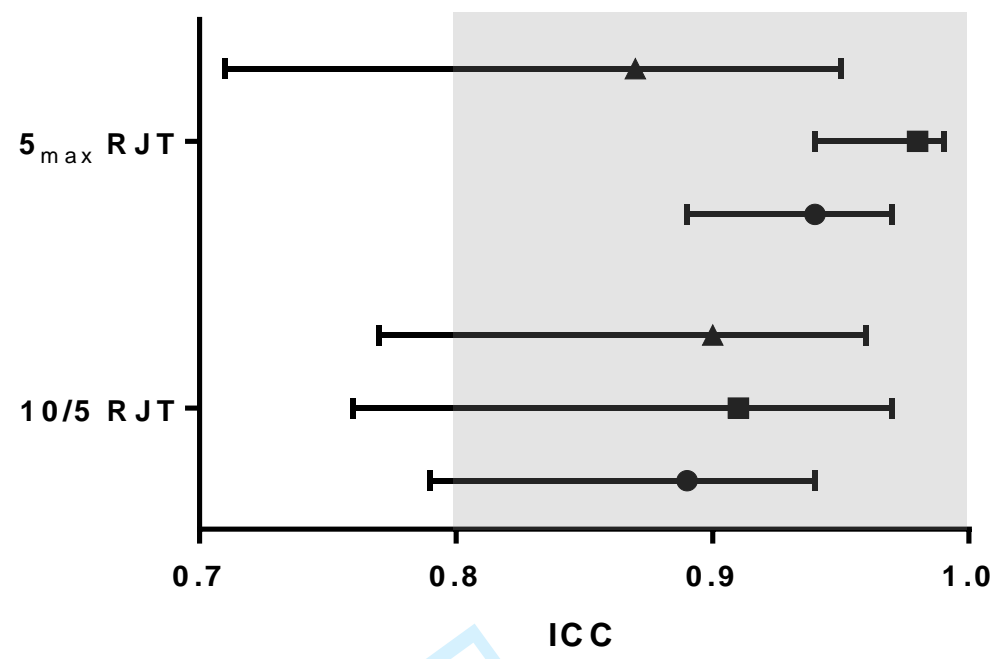

- Males and females

- Females only

- Males only

Figure 1. 


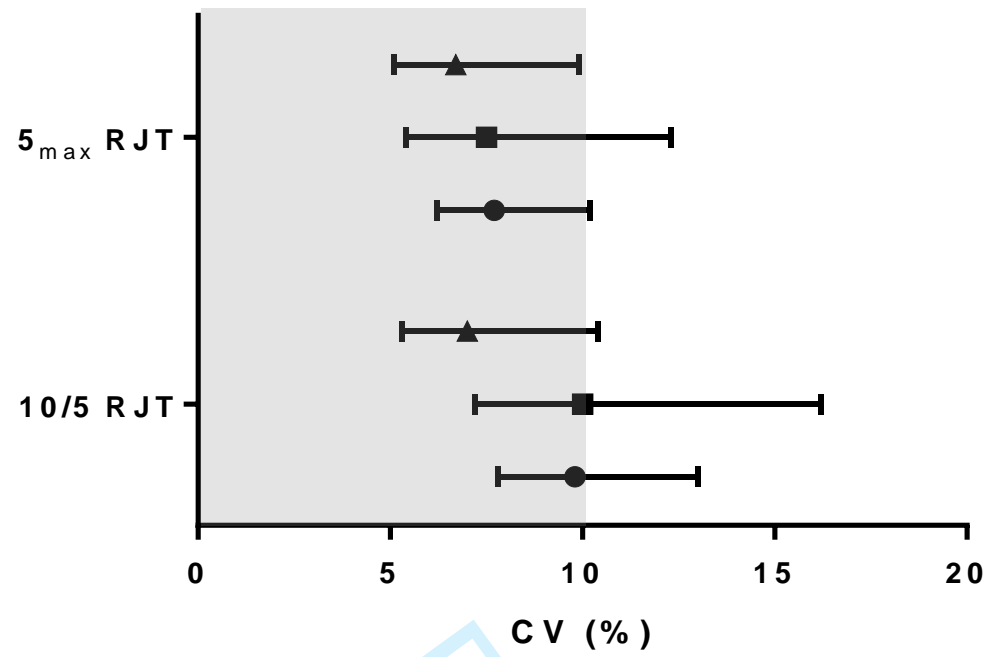

- Males and females

- Females only

$\Delta \quad$ Males only

Figure 2. 
Table 1. Highest RSI (mean \pm SD) for males and females for testing day one and two for both the 10/5 RJT and the $5_{\max }$ RJT

\begin{tabular}{lllll}
\hline Participants & $\begin{array}{l}\mathbf{5}_{\max } \text { RJT-RSI } \\
\text { Day One }\end{array}$ & $\begin{array}{l}\mathbf{5}_{\max } \text { RJT-RSI } \\
\text { Day Two }\end{array}$ & $\begin{array}{l}\text { 10/5 RJT-RSI } \\
\text { Day One }\end{array}$ & $\begin{array}{l}\text { 10/5 RJT-RSI } \\
\text { Day Two }\end{array}$ \\
\hline $\begin{array}{l}\text { Males and } \\
\text { Females }\end{array}$ & $1.39 \pm 0.39$ & $1.43 \pm 0.43$ & $1.38 \pm 0.40$ & $1.40 \pm 0.38$ \\
Males Only & $1.52 \pm 0.26$ & $1.61 \pm 0.29$ & $1.48 \pm 0.31$ & $1.59 \pm 0.29$ \\
Females Only & $1.23 \pm 0.48$ & $1.18 \pm 0.46$ & $1.22 \pm 0.47$ & $1.20 \pm 0.38$ \\
\hline I & & &
\end{tabular}


Table 2. Reliability and usefulness of RSI derived from the 10/5 RJT and $5_{\max }$ RJT for males, females, and males and females combined.

\begin{tabular}{|c|c|c|c|c|c|c|c|c|c|c|c|}
\hline Variable & ICC & $\begin{array}{l}\text { Lower } \\
95 \% \text { CI }\end{array}$ & $\begin{array}{l}\text { Higher } \\
95 \% \text { CI }\end{array}$ & $\mathrm{CV} \%$ & $\begin{array}{l}\text { Lower } \\
95 \% \text { CI }\end{array}$ & $\begin{array}{l}\text { Higher } \\
95 \% \text { CI }\end{array}$ & TE & $\begin{array}{l}\text { SWC } \\
(0.2)\end{array}$ & Rating & $\begin{array}{l}\text { SWC } \\
(0.5)\end{array}$ & Rating \\
\hline $\begin{array}{l}\text { 10/5 RJT-RSI, } \\
\text { males and } \\
\text { females }\end{array}$ & 0.89 & 0.79 & 0.94 & 9.8 & 7.8 & 13.0 & 0.13 & 0.08 & Marginal & 0.19 & Good \\
\hline $\begin{array}{l}\text { 10/5 RJT-RSI, } \\
\text { females }\end{array}$ & 0.91 & 0.76 & 0.97 & 10.0 & 7.2 & 16.2 & 0.14 & 0.09 & Marginal & 0.21 & Good \\
\hline $\begin{array}{l}\text { 10/5 RJT-RSI, } \\
\text { males }\end{array}$ & 0.90 & 0.77 & 0.96 & 7.0 & 5.3 & 10.4 & 0.10 & 0.06 & Marginal & 0.14 & Good \\
\hline $\begin{array}{l}5_{\max } \text { RJT-RSI, } \\
\text { males and } \\
\text { females }\end{array}$ & 0.94 & 0.89 & 0.97 & 7.7 & 6.2 & 10.2 & 0.10 & 0.08 & Marginal & 0.20 & Good \\
\hline $\begin{array}{l}5_{\max } \text { RJT-RSI, } \\
\text { females }\end{array}$ & 0.98 & 0.94 & 0.99 & 7.5 & 5.4 & 12.3 & 0.07 & 0.10 & Good & 0.24 & Good \\
\hline $\begin{array}{l}5_{\max } \text { RJT-RSI, } \\
\text { males }\end{array}$ & 0.87 & 0.71 & 0.95 & 6.7 & 5.1 & 9.9 & 0.10 & 0.06 & Marginal & 0.14 & Good \\
\hline
\end{tabular}

